\title{
Exosome manufacturing status
}

\author{
William Whitford ${ }^{1}$ \& Peter Guterstam*,2 \\ ${ }^{1}$ Strategic Solutions Leader, GE Healthcare, BioProcess, Cell Culture, Logan, UT, USA \\ ${ }^{2}$ Product Manager, GE Healthcare, BioProcess, Downstream Resins, Uppsala, Sweden \\ *Author for correspondence: peter.guterstam@ge.com
}

Exosomes are secreted by mammalian cells and are widely distributed in cellular systems. They are a medium of information and material transmission. The complexity of exosome nature and function is not thoroughly understood. Nevertheless, they are being confirmed as mediators of intercellular communication and play significant roles in many physiological and pathological processes. Significant obstacles to the efficient and robust isolation of large quantities of pure and specific exosomes still exist. These include a lack of understanding of the relationship between exosome characteristics and function, and a shortage of scalable solutions to separate specific exosomes from other large entities remain. Hence, generic production platforms are desired. While solutions suitable for exosome manufacturing under GMP are available, most have been developed for other purposes.

First draft submitted: 24 August 2018; Accepted for publication: 30 January 2019; Published online: 8 July 2019

Keywords: exosome • manufacturing • production

Exosomes are a type of extracellular vesicles (EVs) having a unique generation pathway and characteristic [1]. Microvesicles are formed by outward budding from the plasma membrane, and cells undergoing apoptosis release apoptotic bodies whereas exosomes are generated through a fusion of the plasma membrane with particular endosomal compartments known as multivesicular bodies (Figure 1). The contents of EVs characteristics and cargo vary by the type and state of the cells involved in their generation. They carry such diverse cargo as microRNA, mRNA, lncRNA, DNA, lipids, peptides and vast array of proteins (including oncoproteins, tumor suppressors, transcriptional regulators and splicing factors) [2,3]. An EV classification solely based on their origin (exosomes, microvesicles and apoptotic bodies) is not sufficient to distinguish different EVs. Additional categorization based on morphological characteristics has been suggested [4].

A single definition of exosome subtypes, characteristics and purity has, however, not been universally established. This is because researchers from various disciplines have been approaching them through an evolving understanding of their essential nature, and from different arenas of biotechnology, monitored characteristics and applications. Exosomes are produced by many types of cells and have been discovered in nearly every bodily fluid. Their presence has been reported in the growth medium of many cultured cells, including B lymphocytes, dendritic cells, cytotoxic $\mathrm{T}$ cells, intestinal epithelial cells, neurons, oligodendrocytes, platelets, mast cells and Schwann cells. They have been demonstrated to be active in immune response; neural communication; reproduction and development; as well as in cell proliferation, homeostasis and maturation. Interest in exosomes is growing due to the discovery of their potential in so many research, diagnostic, analytic and therapeutic procedures.

Lately, quite a number of analytical techniques have been applied to their detection, characterization and isolation - with each emphasizing a distinct set of exosome characteristics. Relevant properties for both detection and isolation include morphological characteristics, size, density, $\zeta$ potential and composition - as well as particular chemical and biological characteristics [4,5].

Reasons for interest in the large-scale production of purified lots of exosomes include their observed paracrine-like activity (replacing other communication exhibited by their cells of origin) and their use as a vector of proteins or nucleic acid in therapeutic applications [6-8]. Exosomes have been, for example, suggested as a delivery system for therapeutic compounds - especially as they can pass the blood-brain barrier [9]. 


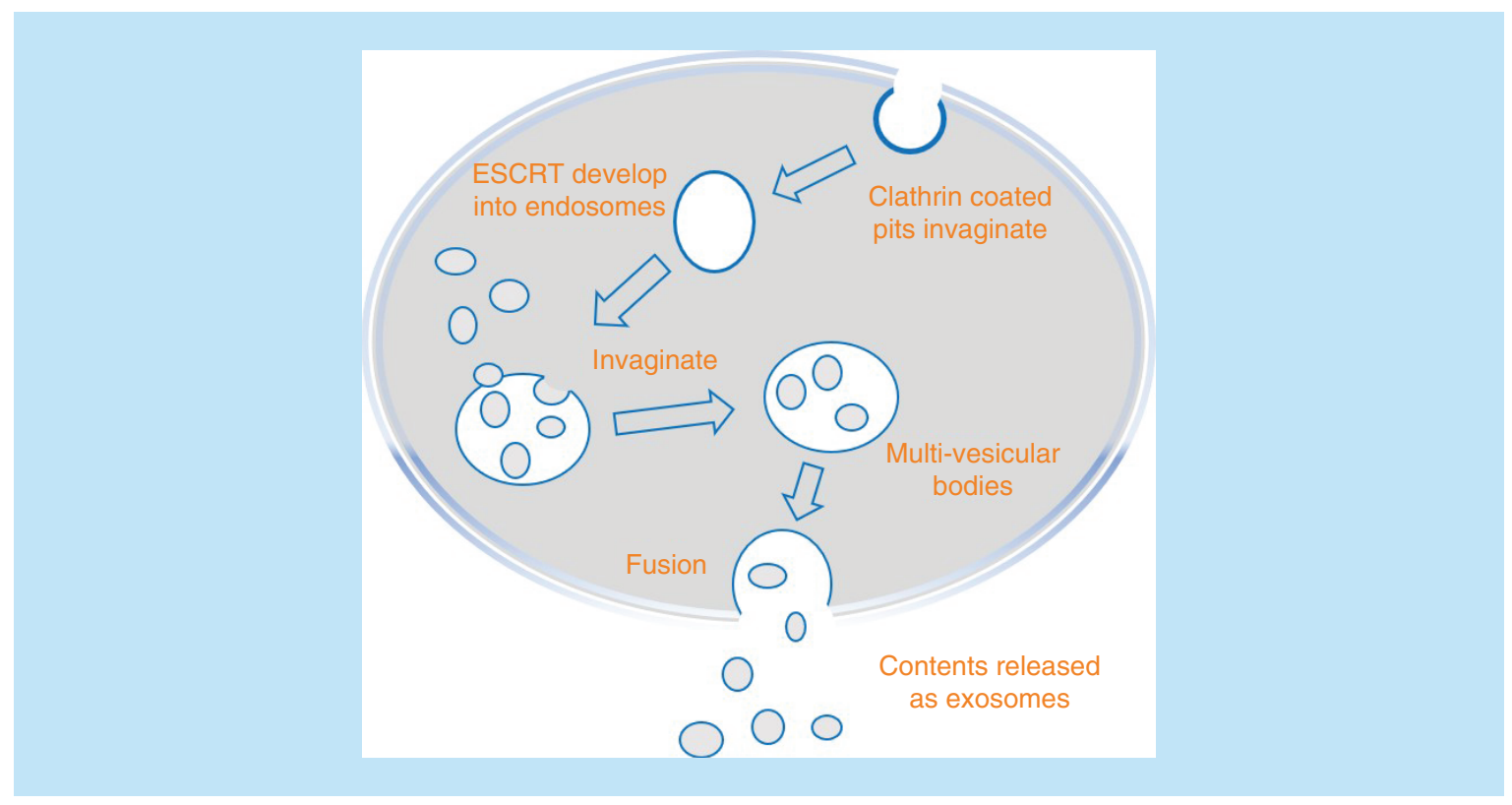

Figure 1. A simplified schematic of exosome biogenesis.

Large-scale production of exosomes is influenced by the specific therapeutic application. For such applications as tissue regeneration and immune response modulation, it is important to produce a therapeutic exosome composition that reflects the expression pattern from the parent cells. The interplay of different exosome subpopulations may have a significant impact upon the therapeutic effect [5]. When using exosomes as therapeutic vectors, such tailored composition of exosome subpopulations may be less crucial.

Isolation and purification of exosomes has, in general, proven to be difficult, and certainly it is even more difficult to isolate a particular subpopulation of exosomes. For example, the use of size-exclusion chromatography, ultrafiltration, precipitation, affinity capture or various centrifugation techniques will greatly increase the concentration and purity of exosome samples, however, no approach alone will produce a lot of highly purified vesicles or separate exosomes from other vesicle types present, and certainly not distinguish between different types of exosomes. A relatively pure population of exosomes can only be achieved through combination of techniques based upon such orthogonal properties as physical size or density and such biochemical parameters as the presence or absence of certain contents. Microfluidic techniques present another very successful category of exosome analysis, segregation and isolation. However, these are not of satisfactory capacity for large-scale production purposes. The therapeutic applications of exosomes, in combination with the delicate challenge to effectively manufacture these new therapeutic entities, motivate a current status update regarding exosome manufacturing alternatives.

\section{Production of exosomes Cellular platforms}

Many cultured cells will secrete exosomes using standard T-flasks and cell culture media. As products of cells, the manufacture of exosomes is dependent on the ability to produce large quantities of cells in ways that do not alter certain cell behavior and characteristics. The potential for changes in cellular phenotype during technical transfer (e.g., scale-up and equipment change) must be considered. As the dynamics of exosome biogenesis is only beginning to be understood, such alterations in any number of characteristics of the cell culture platform might alter the production, composition, attributes or function of the exosomes. The types of cell lines desirable in the manufacturing of exosomes does not aid in this concern. Unlike such platforms as CHO, HeLa or Jurkat lines, it is often necessary to produce exosomes from less established, often primary or stem cell lines [10].

Evidence that alterations in such culture environmental parameters as $\mathrm{pH}$ gradients, mass transfer and hydrodynamic (or shear) force during scale-up can result in changes to such characteristics of the culture platform as induction of apoptotic blebs, downregulation of interleukin receptors, induction of mechanically stimulated protein-kinase pathways and modulation of extracellular signal-related kinases. The consequences of such cellular 
changes upon the exosome product are not well understood [11]. In scaling up, some primary cell lines exhibit a much reduced proliferative capacity, limiting the ultimate culture size, duration, number of production batches or reproducibility. Solutions to this include immortalization by various means, via overexpression of the MYC gene $[12,13]$.

Because many of the cells cultured for exosome research are anchorage-dependent, serum-free media are often not advised, and some degree and schedule of serum supplementation is often required. However, there are reports of protocols for the efficient production by some particular cell types in serum-free, even chemically defined, medium. For example, human-induced pluripotent stem cell-derived cardiovascular progenitors produced exosomes when plated on fibronectin-coated plates and cultured in a serum-free medium supplemented with only an added energy source and bFGF I [14]. Some have reported that shifting from fetal bovine serum (FBS)-containing to serum-free medium resulted in the production of exosomes initially presenting some similar characteristics, while the number of exosomes generated increased. However, further analysis showed that the serum-free exosomes contained different constituent component levels. The serum-free medium appeared to cause a shift in exosomal biology [15].

Exosomes secreted by specific human T-cell clones have been employed to modulate immune cells' activity, including subsets of other T cell. Ceramide, tetraspanins and MAL protein are reported to be critical in T-cell exosome biogenesis. Such molecules may be functional in modulating both exosome production as well as the immune response in therapeutic applications [16]. Many studies have shown that stem cells secrete several important growth factors, and stem cell conditioned medium is often still currently required for efficient stem cell culture in vitro. Another complicating factor in the scale-up of stem cell cultures for exosome manufacturing is the lack of source for large volumes of conditioned medium. It has also been demonstrated that the expression of certain exogenous proteins in cultured cells can influence the type and characteristics of exosomes secreted [17].

Where FBS is required, contamination with bovine exosomes is a concern. To avoid this, exosome-depleted versions of bovine serum are now commercially available. These sera are produced by various exosome-reduction means, with each inducing their own effect upon the serum constituents and characteristics. Furthermore, these sera are affected by the standard source, product, lot specifics and other disparities of normal serum. They do exhibit significant reductions in exosome-sized vesicles resulting in very low levels of CD63-positive cow exosomes or cow miRNAs and vendors provide assay results for such properties as cell culture performance, sterility, mycoplasmas, secretion performance and endotoxins.

Small-scale culture production typically begins with a culture of adherent cells in a flask or plate for a defined period in a standard culture medium. Then, the medium is exchanged for either medium devoid of serum, or medium containing exosome-depleted serum. Many serum-dependent cell cultures will perform relatively normally for a day or two with no serum supplementation. These cultures are then maintained for a defined number of hours or days while they secrete exosomes into this fresh ambient medium. The medium is then decanted and processed for any required vesicle isolation, concentration, characterization and purification.

\section{Production formats \& modes}

Because many of the desired production platforms involve adherent cell culture, scaling-up activities have focused on technologies that maximize culture surface area, such as microcarriers in stirred-tank reactors or culture in fixed-bed or hollow-fiber bioreactors [18]. As mentioned, beyond cell-mass expansion requirements, it is imperative to control identified production environmental parameters such that the cell's phenotype, and therefore secreted exosome characteristics, do not change [19].

As the field of in vitro exosome production is in its infancy, much research and many creative approaches are being reported. Quite a number of the aspects of culture have been examined, including the use of feeder cells, conditioned media, exogenous extracellular matrices and engineered producer-cell transduction. Such approaches may be valuable in some formats for large-scale exosome production. The rational design of biomimetic exosomes is also being addressed.

Methods exist to produce exosome-mimetic vesicles, overcoming such natural limitations as low loading, efficiency and low exosome production yields. One group has developed hybrid exosomes produced through their fusion with liposomes using a freeze-thaw processes [20]. Others describe a virus-mimetic fusogenic exosome demonstrating enhanced fusion efficiency through employing a vascular stomatitis virus viral fusogen within the exosomal membrane [21]. Such methods either produce de novo modified exosomes from engineered cells, or support secondary modification through the fusion of exosomes with liposomes harboring desired proteins, lipids or synthetic polymers. Some have even proposed such creative manufacturing approaches as the in vitro mass production of 
exosome-mimicking nanovesicles using a mini-extruder, and found that they were similar to the native exosomes in some respects [13].

\section{Large-scale production}

Larger-scale batch exosome production is accomplished in such formats as simply employing dozens of large (e.g., T225) flasks, multiple stacked array multilayer culture flasks, large fixed-bed bioreactors, in stirred-tank bioreactors employing microcarriers or continuous production in perfusion reactors [22]. First, it should be mentioned that all the issues of modern technical transfer will exist for development of a large-scale, and especially a clinically relevant, exosome manufacturing format. This includes all the steps involved in shifting from an investigational product to a commercial product, moving from laboratory-scale production to commercial scale and implementing a fileable quality control testing program. It involves such steps as process optimization, confirmation, validation and characterization - as well as process performance qualification activities. Also, pertinent is that many of the newer and higher industry goals in such transfer will apply. These goals include heightened process control, establishing as many closed operations as possible, moving to more automated and 'digital' processes, employing single-use systems, and implementing as many in situ monitoring devices and sample analytics as may be desirable.

Due to the poor efficiency of in vitro exosome production, the current scale-up of standard batch-mode manufacturing can involve hundreds of flasks or a significant investment in the more expensive and complicated multilayer flask systems. Challenges to these approaches include the cost of culture expansion prior to the actual production phase (sometimes involving, e.g., stem cell-conditioned medium) or the additional cost and timing of beginning of the production phase in serum-adjusted or specialty serum-modified medium. Robust production of lot-consistent populations of exosomes necessitates verification of somewhat reproducible culture conditions, which can take time to establish in the scale-up or scale-out of such systems.

Stirred-tank bioreactor cultures employing microcarriers have been reported, as well as a few challenges related to this upstream production approach [19]. There are many scale factors and basic environmental conditions differentiating small-scale flask culture and impeller-based bioreactor culture. Culture progression and production efficiency concerns arise from such factors as cell-to-microcarrier binding, gas mass transfer differences and the hydrodynamic forces generated in sparging and agitation. The effect of these factors upon other such culture characteristics as differentiation, apoptosis potential and exosome product quality and quantity have been reported as well.

Perfusion-based production can alleviate some of these concerns and avoid the added process and limitations associated with microcarriers. Many membrane-based flasks or bioreactors as perfusion-capable technologies have been employed and avoid, or at least reduce, such issues as those associated with hydrodynamic (shear) forces. These systems can not only support culture over an extended period (establishing a continuous biomanufacturing) but can concentrate exosomes within a membrane segregated compartment, facilitating feeding and harvest. In some reactors, the cell-containing side of the perfusion apparatus also supports the segregation of growth factors, allowing a severe reduction in added serum, added factors or conditioned medium. Some platforms have been reported to display entirely new culture characteristics under such high-density culture. These include cell-to-cell contact inhibition of division and a reduction (or elimination) of what had been a constitutive requirement for certain added growth factors or other surface-active agents in the culture medium. However, these systems do not ameliorate all issues of scale-up, -out or of high-density culture effects. For even larger-scale production in a related mode, many porous 3D scaffolds and fiber-based packed- and fixed-bed bioreactors are now available, and some with published applications data.

Hollow-fiber perfusion bioreactors have been employed in cell culture for many years, and their use in large-scale production of exosomes reported. These reactors can support large numbers of cells at high densities in a continuous culture mode without splitting and subculturing of the cells (Figure 2). Cells are cultured on either the inside or outside of the reactor fibers which allow nutrients and waste products to pass through but retain such larger secreted product as exosomes (Figure 3).

In some applications, collection of exosomes can be maintained over several months. In one example, the phenotype of cells in continuous culture remained constant over the 10 -week period of production. It resulted in continuously harvested exosomes which, by orthogonal measure, did not vary in monitored characteristics [23]. So, while many of the large-scale culture methods and technical transfer approaches are similar to protein biological or cells-as-product manufacturing, we see that there are a few exosome-manufacturing-specific phenomena to be considered. 

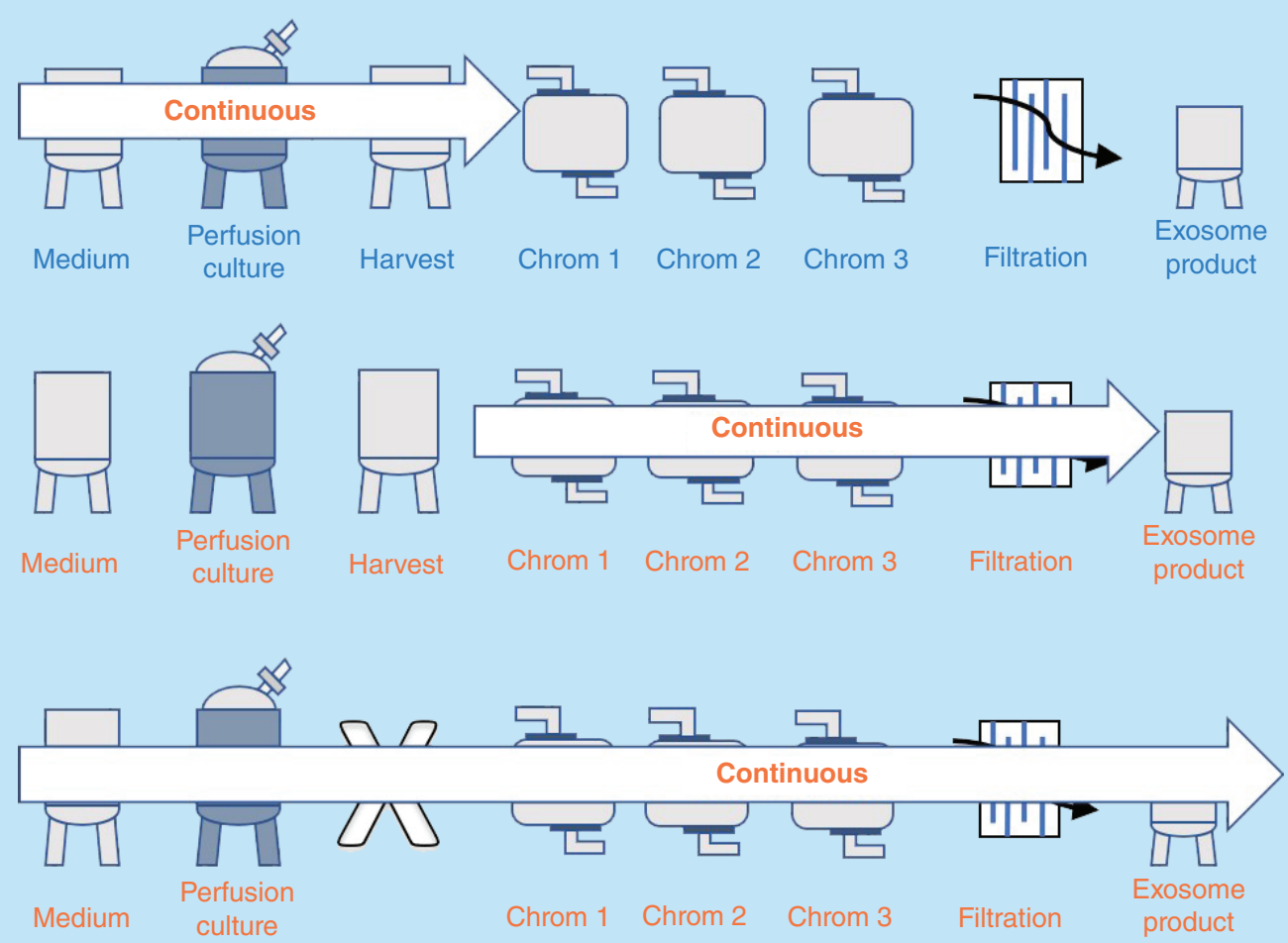

Figure 2. Schematic of model continuous manufacturing approaches.

Figure 3. Cross-section of a hollow fiber reactor. Cells (A) and exosomes (D) in the extracapillary space receive nutrients contained inside (C) the semipermeable hollow

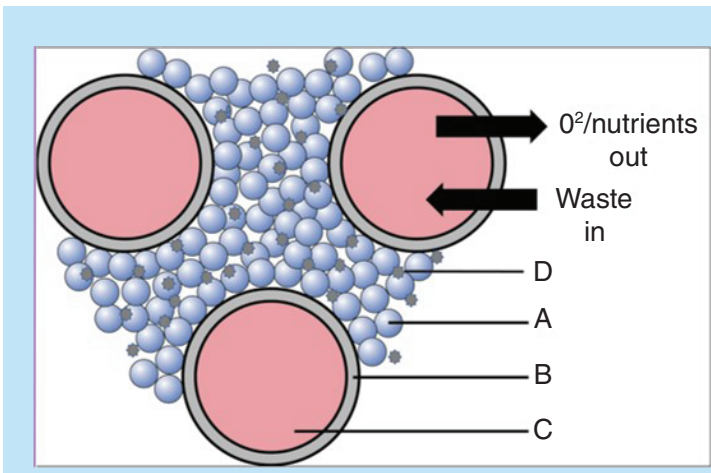
fiber wall (B).

Finally, it should be mentioned that not only are native or innate exosomes employed in biotechnology and medicine, but more recently populations specifically engineered for purpose are being devised. The type of engineering employed ranges from the design of production methods generating exosomes harboring-specific pharmaceutical content, to those producing exosome populations presenting synthetic surface targeting moieties. Generation of exosomes harboring exogenous therapeutic or imaging agents can be accomplished either in vivo or after secretion via several chemical or physical means. Functionalization of the exosome surface ligands for engineered targeting purposes can be accomplished by such approaches as the transfection of the donor cells prior to exosome secretion, or post exosome synthesis through the conjugation of biologically active molecules to harvested exosomes. This latter can be accomplished by click-reactions, such noncovalent chemistries as electrostatic binding, as well as exosome-native transferrin receptor binding [24,25]. 


\section{Isolation \& purification}

When isolating exosomes for therapeutic applications, it is advantageous to have a good understanding of specific characteristics of the target exosome subpopulation or target composition of exosomes. This in combination with robust and consistent upstream feed-stream facilitates the downstream process development. As mentioned in the introduction, three major groups of $\mathrm{EV} s$ have been described according to mechanism of generation: microvesicles, apoptotic bodies and exosomes. The latter are often assumed to represent a homogenous population of EVs. However, recent work has revealed heterogeneities, and the presence of distinct subpopulations of differing biological properties and such molecular compositions as proteomic and nucleic acid repertoires [26]. Such subpopulations have been reported to mediate differential effects on the gene expression programs in recipient cells. Therefore, it must be considered that differing types and scales of isolation techniques may produce different populations of exosome types. For example, separation based on size solely cannot separate the three major EV groups since these three groups are overlapping in size.

Impurities to remove in the production of exosome-based pharmaceutical ingredients are primarily proteins and host cell DNA but also include cell debris, including nonexosome EVs, in other words, apoptotic bodies and microvesicles. Viral clearance and endotoxin removal must also be considered.

Ultracentrifugation is a commonly used approach for isolating EVs [27] but displays such limitations as low yield and modulation or disruption of the target vesicles and it is not suitable for large-scale exosome manufacturing due to technical and practical limitations. The GMP setup processes with several sequential ultracentrifugation steps, generating exceptionally high centrifugal forces (up to $10^{6} \times g$ ) have been described [28]. A slow-speed centrifugation may, however, be considered for mid-scale manufacturing scales, ranging up to a few hundred liters of upstream batch feed. Exosome precipitation is an alternative approach, but such procedures indicate the addition of components that may not be suitable in a large-scale manufacturing setup and imply subsequent removal and associated regulatory and analytical burden $[29,30]$.

Midstream removal of cell debris can be performed based on a sole normal flow filtration $(\sim 5 \mu \mathrm{m})$ step and if needed, be complemented with an initial slow-speed centrifugation. These type of midstream steps are usually robust and effective but have an impact on the yield and on the production cost. After removal of large impurities, it is usually appropriate to concentrate the feed-stream using, for example, ultrafiltration with a $500-750 \mathrm{kD}$ cut-off filter and simultaneously remove remaining large impurities.

Ideally, a specific capture step would be based on a technology that is independent of midstream debris removal. Such capture technologies, based on a very porous base matrix or magnetic beads, however, require specific affinity ligands. Such ligands shall preferably be suitable for sanitization to easily fit in a GMP manufacturing process. With good characterization and analytics of the target exosomes, midstream independent affinity capture opportunities are within reach. Candidates for target exosomes epitopes are CD63, CD9, Tim4. Affinity ligands can also be designed to target recombinantly introduced tags such as the Fc-receptor [31]. Externally displayed epitopes, regardless of whether the origin is endogenous or not, are not only subjects for isolation established upon affinity-based purification, but also subjects for vesicle analysis. Antibodies to such externally displayed epitopes can be immobilized and used as analytical tool, ideally employing surface plasmon resonance-based technologies such as Biacore (GE Healthcare, Uppsala, Sweden) for detection of the target exosome population. Proteins associated with the endosomal sorting complex required for transport (Figure 1), such as ALIX, TSG101 [32] and miRNA, are found in the endosome interior and are therefore candidates for employment as analytical markers of the target exosome subpopulation using other traditional analytical techniques such as mass spectrometry, sequencing, electrophoresis - among others.

In the absence of exosome affinity ligands, separation based on a combination of size and charge are recommended to isolate the target exosome population. Ion-exchange resin, primarily anion exchange, offers a scalable method to isolate the target exosomes from impurities with deviating charge density [33,34]. Variation in the density of glycans present on the exosome surface can impact their separation when using ion-exchange chromatography [34]. Utilizing strong ion-exchange in a bind and elute mode determines elution of the target exosomes at relatively high conductivity. Such a process can, for especially enveloped viruses, have detrimental effect on the exosome yield due to modulation of the vesicle integrity. To minimize yield losses it is therefore important to minimize the process time, especially the critical elution phase of the chromatographic step [35,36]. Chromatographic techniques based on resins that rely upon diffusion of the target entity into the beads are associated with relatively long contact times, due to the limited flow that can be employed. An attractive approach to support higher flow rates and 


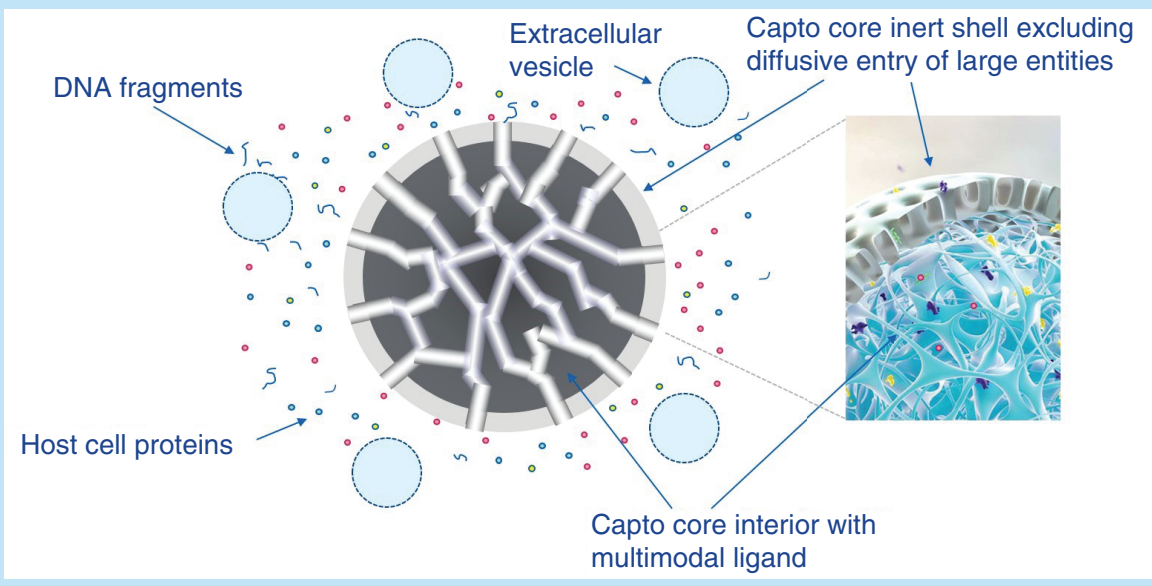

Figure 4. Capto core bead technology principle. Small impurities such as DNA residuals and host cells protein can enter the bead interior by passing the inert shell which has small pore size. The impurities that enter the bead bind almost irreversibly to an octylamine ligand with multimodal functionality. Extracellular vesicles are too big to pass the inert shell and pass in the flow-through.

Reproduced with permission from (c) General Electric Company (2019),

see https://www.gelifesciences.co.jp/catalog/pdf/16188_10061.PDF

thereby shorter contact time and lower yield losses is to use a monolith, membrane or fiber-based chromatographic technique. These allow higher flow as they are independent of allowing the target entity to diffuse into beads [35,37].

Separation solely by charge of the target entity is, in most cases, not satisfactory to generate the desired purity of the target exosome population, and therefore separation based upon size or other properties can be employed to complement separation based on charge [22]. Size separation can be employed before or after an ion-exchange step. The order of these steps must be carefully considered to minimize the need for buffer exchange steps, usually based on ultrafiltration. Another consideration affecting the order of the separation steps is that an ion-exchange step is a good means to reduce potential viral contamination and can provide for endotoxin removal [38].

As the field of exosome manufacturing is so new, a number of creative approaches are appearing. Field-flow fractionation combined with multiangle and dynamic light scattering is being considered a as potentially powerful tool. It combines true size-based separation using field-flow fractionation with an independent determination of size and structure via a light scattering instrument. It can determine highly accurate size distributions and particle concentrations in native solutions. The size fractions may be isolated and automatically collected for further off-line investigation using a standard fraction collector [39].

Size-separation can be effectively performed using chromatographic size-exclusion based on a resin with a pore size that is tailored for the size-interval of the target exosome population. Size-exclusion chromatography is performed with an isocratic flow over a packed resin bed. The bed height must be high, up to $60 \mathrm{~cm}$, to achieve desired resolution. The high bed height in combination with the low sample volume that can be applied in each chromatographic cycle make this size separation technology nonsuitable for large manufacturing. In addition, each chromatographic cycle is long and therefore contributes negatively from a process economy point of view [40].

A more attractive way to remove impurities smaller than the target exosome population is to separate by size, employing a core bead resin. Such resin, for example, Capto Core, is composed of an inactive shell enclosing an activated-ligand core. The inactive shell prohibits large entities from penetrating the smaller pores to the core (Figure 4). Larger entities, in other words, the exosomes, are collected in the flow-through fraction while smaller molecules (such undesired material as DNA, residual host cell proteins and process-related impurities) bind to the resin's active interior ligands. The functionalized core of each bead presents both hydrophobic and positively charged ligands that bind various small contaminants through a highly efficient multimodal chemistry. Different pore size distributions of the inactive shell in combination with flow optimization allow tailoring the size of large entities collected in the flow-through fraction. The beauty of core bead technology is that the bed height can be 
reduced as compared with size-exclusion chromatography and a significantly increased flow rate can be employed. These features are valuable in the manufacturing of exosomes at large scale.

The concentration of intermediate or final preparations often requires variation. This can sometimes be accomplished via adjustment of final steps in the means of collection, isolation or reduced through simple dilution. Concentration of an intermediate or final preparation can be accomplished through a variety of means, including ultracentrifugation to a pellet and resuspension or by density gradient centrifugation in sucrose and band collection, and are reviewed in recent publications [41].

The downstream component of an exosome manufacturing process is usually finalized by a buffer-exchange step and virus filtration. The exact final formulation depends on the application area. Exosomes for therapeutic use may have additional and different process steps. Exosomes with paracrine functionality do not need additional processing, while exosomes used as drug delivery vectors may undergo various loading process steps after the chromatographic isolation [24].

\section{Standards, analytics \& regulatory}

Despite 20 years of research, not only are the basics of exosome biology, occurrence, diversity and identity in their infancy, but also the roles they play in normal cellular and organismal physiology are far from fully understood. Currently, many different analytical techniques have been applied to the identification, quantification and potency evaluation of exosomes - with each measuring one or more of the properties relevant for their detection and isolation mentioned in the Introduction. The field is now populated with a number of immature and empirical descriptions and definitions for a number of reasons. These include an incomplete understanding of the specific characteristics and molecular composition each microvesicle type and subtype, any exosome impurity profile per $s e$, as well as the product and impurity consequences of each exosome harvest source, manufacturing and isolation platform. Standardization and regulatory positions are complicated by the number of proposals for the use of purified preparations of exosomes including diverse research, diagnostic, analytic and product implementation modes and formats (e.g., microfluidic). There is also a wide range of contemplated and proposed therapeutic applications and clinical indications. A further consideration here is that, unlike many other biological entities within the medical applications arena, there exist many specific and distinct tissue and cellular sources (e.g., from cultured cells to autologous or allogeneic primary cells) for exosomes or exosome-containing products, with many having a current set of applicable quality and regulatory considerations. Considering many of upcoming in vitro or companion diagnostic exosome products, it is not clear to the authors which would be properly filed under, for example, a pre-market approval (PMA), 510(k) or humanitarian device exemption (HDE). Regarding the variety of proposed therapeutic products without precedent: depending upon the particular exosome source, isolation approach, described mode of action and route of administration, in the USA it is not intuitively obvious if one would properly file a new drug application (NDA) or biologic license application (BLA) with Center for Drug Evaluation and Research (CDER) or Center for Biologics Evaluation and Research (CBER).

Manufacturers currently work from previously published approaches or design techniques suitable for their current needs. We know of no publications or guidance by national compendial, standards setting or regulatory agencies on the topic. However, as so many highly regulated applications are being pursued for exosomes, these matters have been the topic of significant discussion during international and professional society meetings - and some few have published initial guidelines, suggestions and considerations on the matter [42-44]. Finally, as over a dozen clinical trials of exosome application are ongoing in the USA alone, sufficient evidence of control for investigational new drugs (INDs) have been established, and no doubt NDA filings for these are in final stages of development. For example, the US FDA has recently cleared a stem cell-based IND application for mesenchymal stem cell-derived exosomes as a therapeutic entity for burn victims [45].

\section{Conclusion}

There exist many different production platforms and modes, designed to produce several different types of exosomes, for many divergent applications. Neither standard analytic and isolation techniques been established, nor have standard exosome characterization and subtype definition been achieved. No generic platform or production modes for either the up- or downstream manufacturing of exosomes for any application has been described at this point. Equipment and consumables suitable for exosome manufacturing under GMP are available, but to a great extent these products have been developed for other purposes. Further tailoring and optimization for specific exosome subtypes and needs, within the broader exosome manufacturing arena, is desired. For example, we may 
see perfusion-based upstream manufacturing, harvesting and capture based on midstream independent affinity technologies, and closed processing to remove the need for final virus filtration and associated yield losses.

\section{Future perspective}

Bioproduction is going through a number of revolutions. Uses for exosomes in research, analytic, preclinical testing, diagnostic and therapeutic applications continue to be discovered. Stem and other primary cells are being produced for several US FDA approved trials in clinical applications. As approvals are established, high efficiency, low-risk methods of production will have to be finalized. Both upstream and downstream manufacturing of protein biologicals, vaccines and cells for cell-based therapies are being transformed by such concepts and technologies as single-use, Industry 4.0, factory-of-the-future, modular, continuous and intensified manufacturing initiatives. There is no reason to believe that each of these advances will not influence approaches to both the culture of the cell platforms employed in, as well as the actual, upstream and downstream, production of exosomes themselves.

\section{Executive summary}

- Exosomes are a type of extracellular vesicle of unique generation.

- Their contents are quite diverse and can vary following many factors.

- They naturally occur in nearly every bodily fluid and many cultured cells.

- Interest is due to potential in research, diagnostics, analytics and therapeutics.

- Characterized by morphology, size, density, $\zeta$ potential, composition/properties.

- Large-scale production procedures are influenced by the exosome's application.

- Isolation and purification of exosomes has in general proven to be difficult.

- Pure populations are only achieved through a combination of techniques.

Production of exosomes

- Many cultured cells will secrete exosomes using standard T-flasks and cell culture media.

- It is often necessary to produce exosomes from less established primary or stem cell lines.

- Alterations in culture environmental parameters can affect exosome yield/characteristics.

- Scaling-up from T-flasks can cause issues that have often been addressed in the literature.

- Serum-free culture is discouraged for reasons of attachment and exosome characteristics.

- Successful serum-reduced (or free) culture reported in some cell types/production modes.

- When serum is employed in actual production, reduced-exosome serum is recommended.

- Many factors, metabolites, surface-active proteins, factors implicated in quality production.

- Large-scale: mainly stirred-tank reactor/microcarriers, fixed bed or hollow fiber bioreactors.

- Culture methods: feeder cells, conditioned media, extracellular matrices, engineered cells.

- Exosomes method: vesicle and virus-mimetic, various hybrid-fusion, mini-extruder effected.

Large-scale production

- Formats: T-225 flasks, multilayer flasks, large fixed-bed reactors, microcarriers in stirred-tank reactor.

- All issues of modern technical transfer will exist for development of large-scale manufacturing format.

- Concerns arise from, for example, cells-to-microcarrier binding, gas mass-transfer and the shear forces.

- Perfusion-based bioproduction can alleviate many bioreactor culture and technology transfer concerns.

- Many membrane-based flasks or bioreactors as perfusion-capable technologies have been employed.

- Perfusion supports culture over an extended period of time and yields concentrated lots of exosomes.

- Many porous 3D scaffolds, fiber-based packed- and fixed-bed bioreactors have been adapted for use.

- Hollow-fiber perfusion bioreactors are now being successfully used in large-scale exosome production.

- Perfusion supports high density, continuous culture, reduced or eliminated serum/factor requirement.

- Exosomes specifically engineered for research, diagnostic or therapeutic purposes are being designed.

- From harboring pharmaceutical content, to populations presenting synthetic surface targeting moieties.

- Exosomes with exogenous therapeutic or imaging agent cargo produced via chemical or physical ways.

Isolation \& purification

- Scalable solutions exist that support regulated therapeutic applications.

- Support for bioreactor harvest from batch- or perfusion-based upstream manufacturing.

- Filter-based removal of cell debris and large-sized process impurities.

- Isolation and purification based on bind/elute chromatography and size-specific separation.

- Polishing methods to control impurities. 
Standards, analytic \& regulatory

- Affected by that exosome biology, occurrence, diversity and identity are in their infancy.

- Many analytical techniques have been applied to the identification, quantification and potency.

- Immature and empirical descriptions, definitions because of ill-defined characteristics, molecular composition.

- Standardization and regulatory positions are complicated by the number and diversity of exosome considerations.

- Including sources, isolation and processing approaches, described mode of action and route of administration.

- Many diverse research, diagnostic, analytic and therapeutic product implementation modes/formats exist.

- No publications or guidance by national compendial, standards setting or regulatory agencies on the topic.

- US FDA has cleared stem cell-based investigational new drug applications for mesenchymal stem cell-derived exosomes as therapeutic entities.

Conclusion

- Neither standard analytic nor isolation techniques have been established.

- No standard exosome characterization and subtype definition have been achieved.

- No generic platform or production modes for either up- or downstream manufacturing.

- Equipment and consumables suitable for exosome manufacturing under GMP are available.

- Different production platforms and modes are designed to produce different types of exosomes.

Future perspective

- More research, analytic, preclinical testing, diagnostic and therapeutic applications are yet to be discovered.

- Future seeing single-use, Industry 4.0, factory-of-the-future, modular, continuous and intensified approaches.

Financial \& competing interests disclosure

Whitford W and Guterstam P are both employed by GE Healthcare a supplier of equipment and consumables for the manufacturing of biological therapeutics. The authors have no other relevant affiliations or financial involvement with any organization or entity with a financial interest in or financial conflict with the subject matter or materials discussed in the manuscript apart from those disclosed.

No writing assistance was utilized in the production of this manuscript.

\section{Open access}

This work is licensed under the Attribution-NonCommercial-NoDerivatives 4.0 Unported License. To view a copy of this license, visit http://creativecommons.org/licenses/by-nc-nd/4.0/

\section{References}

Papers of special note have been highlighted as: $\bullet$ of interest

1. Raposo G, Stoorvogel W. Extracellular vesicles: exosomes, microvesicles and friends. J. Cell Biol. 200(4), 373-383 (2013).

2. Valadi H, Ekstrom K, Bossios A, Sjostrand M, Lee JJ, Lotvall JO. Exosome-mediated transfer of mRNAs and microRNAs is a novel mechanism of genetic exchange between cells. Nat. Cell Biol. 9(6), 654-659 (2007).

- A door opener to understand the width of exosome-mediated communication.

3. Xu R, Greening DW, Zhu HJ, Takahashi N, Simpson RJ. Extracellular vesicle isolation and characterization: toward clinical application. J. Clin. Invest. 126(4), 1152-1162 (2016).

4. Zabeo D, Cvjetkovic A, Lasser C, Schorb M, Lotvall J, Hoog JL. Exosomes purified from a single cell type have diverse morphology. J. Extracell. Vesicles 6(1), 1329476 (2017).

5. van der Pol E, Hoekstra AG, Sturk A, Otto C, van Leeuwen TG, Nieuwland R. Optical and nonoptical methods for detection and characterization of microparticles and exosomes. J. Thromb. Haemost. 8(12), 2596-2607 (2010).

6. El Harane N, Kervadec A, Bellamy V et al. Acellular therapeutic approach for heart failure: in vitro production of extracellular vesicles from human cardiovascular progenitors. Eur. Heart J. 39(20), 1835-1847 (2018).

7. Herrera MB, Fonsato V, Gatti S et al. Human liver stem cell-derived microvesicles accelerate hepatic regeneration in hepatectomized rats. J. Cell Mol. Med. 14(6B), 1605-1618 (2010).

8. Zhang J, Guan J, Niu X et al. Exosomes released from human-induced pluripotent stem cells-derived MSCs facilitate cutaneous wound healing by promoting collagen synthesis and angiogenesis. J. Transl. Med. 13, 49 (2015).

9. Matsumoto J, Stewart T, Banks WA, Zhang J. The transport mechanism of extracellular vesicles at the blood-brain barrier. Curr. Pharm. Des. 23(40), 6206-6214 (2017).

10. Cheng L, Zhang K, Wu S, Cui M, Xu T. Focus on mesenchymal stem cell-derived exosomes: opportunities and challenges in cell-free therapy. Stem Cells Int. 2017, 6305295 (2017). 
11. Brindley D, Moorthy K, Lee JH, Mason C, Kim HW, Wall I. Bioprocess forces and their impact on cell behavior: implications for bone regeneration therapy. J. Tissue Eng. 2011, 620247 (2011).

12. Chen TS, Arslan F, Yin Y et al. Enabling a robust scalable manufacturing process for therapeutic exosomes through oncogenic immortalization of human ESC-derived MSCs. J. Transl. Med. 9, 47 (2011).

13. Chen TS, Wee Yeh Yeo R, Arslan F et al. Efficiency of exosome production correlates inversely with the developmental maturity of MSC donor. J. Stem Cell Res. Ther. 3(145), 1-10 (2013).

14. Preet R, Zhuang S, Hung WT, Christenson L, Dixon DA. The RNA binding protein HuR enhances exosome secretion in colorectal cancer. Gastroenterology 150(4) S27 (2016).

15. Li J, Lee Y, Johansson HJ et al. Serum-free culture alters the quantity and protein composition of neuroblastoma-derived extracellular vesicles. J. Extracell. Vesicles 4, 26883 (2015).

16. Ventimiglia LN, Alonso MA. Biogenesis and function of T cell-derived exosomes. Front. Cell Dev. Biol. 4, 84 (2016).

17. Phan J. Modification of the Extracellular Environment of Placenta-Derived Mesenchymal Stromal Cells for Improved Exosome Production [PhD Thesis]. California State University, Sacramento, CA, USA (2018).

18. Panchalingam KM, Jung S, Rosenberg L, Behie LA. Bioprocessing strategies for the large-scale production of human mesenchymal stem cells: a review. Stem Cell Res. Ther. 6, 225 (2015).

19. Chen AK, Chen X, Choo AB, Reuveny S, Oh SK. Critical microcarrier properties affecting the expansion of undifferentiated human embryonic stem cells. Stem Cell Res. 7(2), 97-111 (2011).

20. Sato YT, Umezaki K, Sawada S et al. Engineering hybrid exosomes by membrane fusion with liposomes. Sci. Rep. 6, 21933 (2016).

21. Yang Y, Hong Y, Nam GH, Chung JH, Koh E, Kim IS. Virus-mimetic fusogenic exosomes for direct delivery of integral membrane proteins to target cell membranes. Adv.Mater. 29(13) doi: 10.1002/adma.201605604 (2017).

22. Colao IL, Corteling R, Bracewell D, Wall I. Manufacturing exosomes: a promising therapeutic platform. Trends Mol. Med. 24(3), 242-256 (2018).

- A good introduction to the uses, production, purification and characterization of exosomes.

23. Whitford WG, Ludlow JW, Cadwell JJS. Continuous production of exosomes. Genet. Eng. News 35(16) 34 (2015).

- A good early example of efficient high-quantity hollow-fiber production of exosomes.

24. Luan X, Sansanaphongpricha K, Myers I, Chen H, Yuan H, Sun D. Engineering exosomes as refined biological nanoplatforms for drug delivery. Acta Pharmacol. Sin. 38(6), 754-763 (2017).

25. Willis GR, Kourembanas S, Mitsialis SA. Toward exosome-based therapeutics: isolation, heterogeneity, and fit-for-purpose potency. Front. Cardiovasc. Med. 4, 63 (2017).

26. Willms E, Johansson HJ, Mager I et al. Cells release subpopulations of exosomes with distinct molecular and biological properties. Sci. Rep. 6, 22519 (2016).

27. Greening DW, Xu R, Ji H, Tauro BJ, Simpson RJ. A protocol for exosome isolation and characterization: evaluation of ultracentrifugation, density-gradient separation, and immunoaffinity capture methods. Methods Mol. Biol. 1295, 179-209 (2015).

28. Li P, Kaslan M, Lee SH, Yao J, Gao Z. Progress in exosome isolation techniques. Theranostics 7(3), 789-804 (2017).

29. Gardiner C, Di Vizio D, Sahoo S et al. Techniques used for the isolation and characterization of extracellular vesicles: results of a worldwide survey. J. Extracell. Vesicles 5, 32945 (2016).

- A useful survey of the current practices for the isolation, purification and characterization of extracellular vesicles.

30. Hill AF, Pegtel DM, Lambertz U et al. ISEV position paper: extracellular vesicle RNA analysis and bioinformatics. J. Extracell. Vesicles 2 doi: 10.3402/jev.v2i0.22859 (2013) (Epub ahead of print).

31. Wiklander O, Görgens A, Gupta D: EP3487529 (2018).

32. Raiborg C, Stenmark H. The ESCRT machinery in endosomal sorting of ubiquitylated membrane proteins. Nature 458(7237), 445-452 (2009).

33. Kim DK, Nishida H, An SY, Shetty AK, Bartosh TJ, Prockop DJ. Chromatographically isolated CD $63^{+} \mathrm{CD} 81^{+}$extracellular vesicles from mesenchymal stromal cells rescue cognitive impairments after TBI. Proc. Natl Acad. Sci. USA 113(1), 170-175 (2016).

34. Kosanovic M, Milutinovic B, Goc S, Mitic N, Jankovic M. Ion-exchange chromatography purification of extracellular vesicles. Biotechniques 63(2), 65-71 (2017).

35. McNally DJ, Darling D, Farzaneh F, Levison PR, Slater NK. Optimised concentration and purification of retroviruses using membrane chromatography. J. Chromatogr. A 1340, 24-32 (2014).

36. Rodrigues T, Carvalho A, Roldao A, Carrondo MJ, Alves PM, Cruz PE. Screening anion-exchange chromatographic matrices for isolation of onco-retroviral vectors. J. Chromatogr. B Analyt. Technol. BioMed Life Sci. 837(1-2), 59-68 (2006).

37. Burden CS, Jin J, Podgornik A, Bracewell DG. A monolith purification process for virus-like particles from yeast homogenate. $J$ Chromatogr. B Analyt. Technol. BioMed Life Sci. 880(1), 82-89 (2012). 
38. Jagschies G, Lacki K. Biopharmaceutical processing, process capability requirements. In: Biopharmaceutical Processing. Jagschies G, Lindskog E, Łącki KM, Galliher P (Eds.) Elsevier Radarweg, Amsterdam, The Netherlands (2018).

39. Zhang H, Freitas D, Kim HS et al. Identification of distinct nanoparticles and subsets of extracellular vesicles by asymmetric flow field-flow fractionation. Nat. Cell Biol. 20(3), 332-343 (2018).

40. Manzari RC, O’Donnell JK. SEC in the modern downstream purification process. BioPharm Int. 28(3), 34-37 (2015).

41. Konoshenko MY, Lekchnov EA, Vlassov AV, Laktionov PP. Isolation of extracellular vesicles: general methodologies and latest trends. BioMedRes. Int. 2018, 8545347 (2018).

42. Bersenev A. Considerations for clinical translation of extracellular vesicles as new class of therapeutics. In: Stem Cell Assays. William Gunn, Alexey Bersenev (Eds). Department of Hematology Children's Hospital of Philadelphia, PA, USA (2016).

- Describes EV as an evolving new class of therapeutics with unclear regulatory pathways, manufacturing standards and quality controls. A review of the International Society for Extracellular Vesicles (ISEV) position paper.

43. Lener T, Gimona M, Aigner L et al. Applying extracellular vesicles-based therapeutics in clinical trials - an ISEV position paper. J. Extracell. Vesicles 4, 30087 (2015).

44. Vogel R, Coumans FA, Maltesen RG et al. A standardized method to determine the concentration of extracellular vesicles using tunable resistive pulse sensing. J. Extracell.Vesicles 5, 31242 (2016).

45. Exosome RNA Administrator. Aegle therapeutics receives IND clearance from FDA for stem cell-derived extracellular vesicle therapy (2018). www.exosome-rna.com/aegle-therapeutics-receives-ind-clearance-from-fda-for-stem-cell-derived-extracellular-vesicle -therapy/ 